\title{
SOBRECARGA DE CUIDADORES INFORMAIS DE IDOSOS
}

Gabriela Maçarico Figueiredo Garcia ${ }^{1}$ Estudante de Medicina, Universidade Cesumar, Maringá (PR). E-mail:

gabimacarico@hotmail.com

\section{Priscila Hitomi Imoto}

Estudante de Medicina,

Universidade Cesumar, Maringá

(PR). E-mail:

pri.imoto@hotmail.com

\section{Marcelo Picinin Bernuci ${ }^{3}$}

Doutorado em Fisiologia pela

Faculdade de Medicina de

Ribeirão Preto-Universidade de

São Paulo (SP). Docente

Permanente do Programa de Pós-

graduação em Promoção da

Saúde e do Curso de Medicina da

Unicesumar - Universidade

Cesumar. Coordenador da

Comissão de Ética no Uso de

Animais da Unicesumar.

Pesquisador do Instituto Cesumar

de Ciência, Tecnologia e Inovação

- ICETI. E-mail:

mbernuci@gmail.com

\section{Mirian Ueda}

Yamagucchi ${ }^{4}$

Doutorado em Ciências

Farmacêuticas (2009) pela

Universidade Estadual de Maringá

(PR). Docente Permanente do

Programa de Pós-graduação em

Promoção da Saúde e do Curso de Medicina da Unicesumar -

Universidade Cesumar.

Pesquisadora do Instituto Cesumar de Ciência, Tecnologia e Inovação - ICETI. E-mail:

mirianueda@gmail.com

\section{Ely Mitie Massuda ${ }^{5}$}

Doutorado em História Econômica pela Universidade de São Paulo

(SP). Docente Permanente do

Programa de Pós-graduação em

Promoção da Saúde e do

Programa de Pós-graduação em

Gestão do Conhecimento nas

Organizações. Pesquisadora do

Instituto Cesumar de Ciência,

Tecnologia e Inovação - ICETI. E-

mail: elymiitie.m@gmail.com

\section{INFORMAL CAREGIVERS OVERLOAD OF ELDERLY}

\section{RESUMO}

Cuidadores, muitas vezes, sofrem restrições em suas vidas devido à necessidade de dedicação ao cuidado direto ao idoso. O objetivo da pesquisa consistiu em analisar a percepção de sobrecarga de trabalho de cuidadores informais de idosos e sua associação com fatores socioeconômicos. Estudo descritivo transversal, de abordagem quantitativa e qualitativa, a amostra foi do tipo não probabilística $(n=20)$, desenvolvida com os cuidadores de idosos vinculados à Estratégia Saúde da Família de uma Unidade Básica de Saúde (UBS) do município de Maringá, Paraná, Brasil. A coleta de dados ocorreu de fevereiro a junho de 2017, com os seguintes instrumentos: questionário sociodemográfico, Escala Zarit Burden Interview - EZBI e questionário com questões abertas. Para avaliar as associações utilizaram-se os coeficientes de correlação de Pearson e Somer. Todos os cuidadores entrevistados eram do sexo feminino, sendo a maioria filhas ou esposas dos idosos, com escolaridade igual ou inferior ao ensino médio, renda de 1 a 2 salários mínimos e idade superior a 51 anos. $50 \%$ dos cuidadores apresentaram sobrecarga moderada, $25 \%$ apresentaram sobrecarga moderada/severa e $25 \%$, sobrecarga severa. Os fatores associados com a percepção de sobrecarga foram: faixa etária, escolaridade, renda e grau de parentesco. A análise qualitativa das respostas mostra que a percepção de sobrecarga de trabalho é amenizada pelos laços afetivos entre os cuidadores e os idosos. A percepção de sobrecarga de trabalho de cuidadores de idosos é influenciada por fatores socioeconômicos e demográficos bem como pelas relações interpessoais estabelecidas no ato do cuidar.

PALAVRAS-CHAVE: Cuidadores familiares; envelhecimento; saúde ocupacional. 


\section{ABSTRACT}

Caregivers often endure restrictions in their lives due to the need for dedication to direct care to the elderly. The aim of this research was to analyze the perception of work overload of informal caregivers of the elderly and the association between socioeconomic variables. A cross-sectional descriptive study, with a qualitative and quantitative approach, the sample was nonprobabilistic $(n=20)$, developed with caregivers of elderly people linked to the Family Health Strategy of a Basic Health Unit of Maringá, Paraná, Brazil. Data collection took place from February to June 2017, with the following instruments: sociodemographic questionnaire, Zarit Burden Interview Scale - EZBI and analysis of interview content. Pearson and Somer correlation coefficients were used to evaluate the associations. All the caregivers interviewed were female, with the majority being daughters or wives of the elderly, with education equal to or less than high school, income of 1 to 2 minimum wages and age over 51 years. $50 \%$ of the caregivers presented moderate overload, $25 \%$ presented moderate / severe overload and $25 \%$ presented severe overload. Factors associated with the perception of overload were: age, schooling, income and kinship. The qualitative analysis of the answers shows that the perception of work overload is softened by affective ties between caregivers and the elderly. The perception of work overload of elderly caregivers is influenced by socioeconomic and demographic factors and by the interpersonal relationships established in the act of caring.

Keywords: Caregivers. Aging. Occupational health.

\section{INTRODUC̣̃̃O}

O envelhecimento populacional tem ocorrido rapidamente como resultado de uma melhora na oferta de serviços de saúde, propagação de hábitos saudáveis, aumento da expectativa de vida e diminuição da fertilidade (SINGER, 2016; SANDERSON; SCHERBOV; GERLAND, 2017). De acordo com relatório divulgado pela Organização Mundial da Saúde (OMS), 11\% da população mundial tem mais de 60 anos de idade, com um aumento projetado, até 2050, para 22\% (WHO, 2015). Esta rápida transição demográfica acarreta implicações significativas para o sistema de saúde, o que tem evocado ações globais para o enfrentamento do problema (WHO, 2017). No entanto, dado o grande contingente de pessoas que irão lidar com os eventos naturais do processo de envelhecimento, mais investigação e atenção política sobre os determinantes da qualidade de vida dessa população serão necessários.

As repercussões negativas do envelhecimento, marcadas pela maior frequência de doenças crônicas não transmissíveis e perda da capacidade funcional (WANG et al, 2017; ABAJOBIR, et al, 2017) emerge de maneira paradoxal a relação entre expectativa de vida e qualidade de vida. Esse paradoxo gera preocupações quanto à capacidade dos sistemas sociais para atender à demanda crescente por cuidados em função do aumento das limitações funcionais, abrindo um espaço profícuo para atuação dos cuidadores, sejam eles formais ou informais (ALSAEED et al., 2016; TKATCH et al., 2016; COMMISSO et al., 2017). No Brasil, segundo dados da Pesquisa Nacional de Saúde de 2013, o cuidado informal é a principal fonte de ajuda domiciliar aos idosos com limitações funcionai (LIMA-COSTA et al., 2017), embora haja registros de que 
muitos idosos, especialmente aqueles economicamente desfavorecidos, ainda careçam desta atenção (LIMA-COSTA et al., 2016). É neste contexto que o papel do cuidador informal assume relevância, uma vez que as repercussões de sua atuação estão diretamente relacionadas com o bem-estar do idoso.

A oficialização da atividade de atuação dos cuidadores tem sido pautada no Brasil diante de intenso debate (BATISTA; ALMEIDA; LANCMAN, 2014; DEBERT; OLIVEIRA, 2015). Com a promulgação da Política Nacional do Idoso em 1999, a definição de cuidador foi estabelecida, descrita como uma pessoa que, com ou sem remuneração, realiza o cuidado do idoso dependente ou doente (BRASIL, 2006). A fim de subsidiar diretrizes para programas de capacitação de cuidadores, a Portaria Interministerial $n \times 5.153 / 99$, instituiu 0 Programa Nacional de Cuidadores de Idosos (BRASIL, 1999). Em 2008, a Secretaria Especial dos Direitos Humanos publicou o Manual do Cuidador da Pessoa Idosa (BORN, 2008), destacando a necessidade do autocuidado do cuidador.

De fato, o ato de cuidar de idosos envolve sobreposição de tarefas, diminuição do tempo livre, cansaço, exigências e expectativas que podem comprometer a qualidade de vida do cuidador (LÓPEZ-MARTÍNEZ; FRÍAS-OSUNA; DEL-PINOCASADO, 2017; DEL-PINO-CASADO et al., 2018). Os problemas físicos, psíquicos e sociais associados a sobrecarga de trabalho prejudicam sobremaneira a saúde do cuidador (OLDENKAMP et al. 2016; FREDERICK, 2018; FEKETE et al., 2017; PENDERGRASS, 2018) e interferem na dinâmica do ato de cuidar (OLDENKAMP et al., 2017; LAMBERT, et al, 2017). Embora muitos esforços têm se direcionado para a amenizar os efeitos da sobrecarga de trabalho do cuidador de idosos (MADARA, 2016; CHEN et al., 2017; TKATCH et al., 2017), no Brasil, poucos estudos têm se dedicado para essa temática (MOTA et al., 2015; MONTEIRO: MAZIN; DANTAS, 2015; MUNIZ, et al., 2016; SILVA LEITE et al., 2017; KOYANAGI et al., 2018)

Diante deste cenário, o presente estudo buscou avaliar percepção de sobrecarga de trabalho do cuidador informal de idosos e sua associação com variáveis socioeconômicas bem como analisar as reflexões do cuidador acerca de seu encargo.

\section{MÉTODOS}

Estudo de caráter descritivo exploratório, de corte transversal, abordagem quantitativa e qualitativa, foi realizado junto a cuidadores informais de idosos vinculados às Equipes de Saúde da Família de uma Unidade Básica de Saúde (UBS) do município de Maringá (PR). A pesquisa foi realizada com base no número de idosos com dependência cognitiva e/ou funcional cadastrados em três das Equipes de Saúde da Família (ESF) que dispunham de informação sobre o número de cuidadores informais que prestam cuidados domiciliares a idosos dependentes. As demais ESF da referida UBS não possuíam esses dados.

A busca dos sujeitos foi realizada por meio de prontuários dos idosos dependentes acompanhados na unidade de atenção básica à saúde e por informações colhidas junto às agentes comunitárias de saúde das ESF totalizando 36 participantes. Foram excluídos idosos dependentes que não contavam com cuidador e cuidadores ausentes após três tentativas de contato presenciais, efetivando-se cuidadores de 20 idosos. Os contatos foram estabelecidos com os cuidadores, presencialmente, pelas pesquisadoras no domicílio dos idosos. Entre os convidados não houve nenhuma recusa de 
participação. A aplicação do instrumento de coleta de dados e do questionário com questões abertas foi realizada pelas pesquisadoras, sem a presença das agentes comunitárias de saúde, no domicílio dos idosos. O tempo médio para a aplicação dos instrumentos de pesquisa foi de 100 minutos.

$\mathrm{O}$ instrumento de coleta de dados foi aplicado através de uma entrevista, sendo este dividido em: dados de identificação, aspectos sociodemográficos e a Escala Zarit Burden Interview - EZBI (SCAZUFCA, 2002), traduzida e validada no Brasil para avaliação da sobrecarga de cuidadores. A EZBI consiste em uma série de afirmativas que permitem avaliar como as pessoas se sentem ao cuidar de outra pessoa. As afirmativas estão relacionadas aos sentimentos algumas vezes despertados ao cuidar do idoso como o estresse, a tensão, a irritabilidade e a vergonha pelo comportamento do mesmo, além de questões sobre alguns anseios como o receio pelo futuro do idoso e a sensação de incapacidade de cuidar por muito mais tempo. São também abordadas questões financeiras como a falta de dinheiro para cuidar do idoso, somada a outras despesas. Outro aspecto também avaliado é a interferência na vida social, nos relacionamentos e na privacidade dos cuidadores, além da sensação de perda de controle da própria vida, da piora na saúde e do sentimento de sobrecarga.

O EZBI é composto por 22 itens, cujas respostas variam de 0 a 4, em escala de Likert, sendo: $(0$ = nunca, 1 = raramente, 2 = algumas vezes, 3 = frequentemente e 4 = sempre). A exceção é o último item, em que o pesquisado é questionado se está se sentindo sobrecarregado por exercer a função de cuidador: $(0=$ nem um pouco, 1 = um pouco, 2 = moderadamente, 3 = muito, 4 = extremamente). O escore da escala varia de 0 a 88, sendo que quanto maior a pontuação, maior é a sobrecarga. Tal sobrecarga pode ser classificada, de acordo com o score estabelecido por Zarit e Zarit (1987), em pequena (0 a 20 pontos), moderada (21 a 40 pontos), moderada a severa ( 41 a 60 pontos) e severa ( 61 a 88 pontos).

$\mathrm{Na}$ mesma ocasião da aplicação do instrumento de coleta de dados, um questionário com questões abertas foi conduzido para que o cuidador descreva sua realidade, seus sentimentos, suas dificuldades e anseios. As entrevistas foram gravadas, com autorização dos participantes e, posteriormente transcritas pelos autores do artigo.

Uma análise descritiva dos resultados foi realizada para a obtenção de gráficos e tabelas de frequência, com o intuito de caracterizar os indivíduos. Para descrição dos resultados foram utilizadas a frequência absoluta e a porcentagem para as variáveis categóricas. Já os escores do EZBI foram descritos pela média aritmética simples, desvio padrão, coeficiente de variação, mínimo, máximo e amplitude. Por fim, foram construídos diagramas de dispersão para avaliar a relação do escore do EZBI com as variáveis sociodemográficas.

Para avaliar a relação entre o escore do EZBI com a idade, escolaridade e renda (variáveis ordinais) utilizou-se o coeficiente de correlação por postos de Spearman. Já para examinar a relação entre o escore do EZBI com o estado civil e grau de parentesco (considerando apenas as duas categorias mais frequentes de cada variável), a estatística utilizada foi o coeficiente rank biserial correlation também conhecida como $\mathrm{D}$ de Somer, adequado para a mensuração da correlação entre um escore com uma variável dicotômica. Ambos coeficientes variam no intervalo de $(-1,1)$.

Todas as análises foram realizadas com o auxílio do ambiente estatístico $R(R$ Development Core Team), versão 3.3.1.

O projeto de pesquisa foi aprovado pelo Assessoria de Formação e Capacitação Permanente dos Trabalhadores de Saúde (CECAPS), órgão que regulamenta as pesquisas a serem realizadas nas Unidades Básicas de Saúde em Maringá. 
Posteriormente, foi submetido ao Comitê de Ética em Pesquisa (CEP) da Unicesumar e aprovado sob o parecer 2007415.

Para a execução do presente projeto, após a orientação sobre os objetivos da pesquisa e direitos do participante, foi solicitada aos sujeitos do estudo a assinatura do Termo de Consentimento Livre e Esclarecido (TCLE). Os cuidadores e dos idosos foram citados nas entrevistas por iniciais para preservar o anonimato dos mesmos.

\section{RESULTADOS}

Os dados referentes a caracterização socioeconômica e demográfica dos cuidadores informais de idosos encontram-se organizados na Tabela 1.

Tabela 1 - Distribuição de frequências das variáveis socioeconômicas e demográficas dos cuidadores participantes da pesquiss.

\begin{tabular}{|c|c|c|}
\hline & Frequência & $\%$ \\
\hline \multicolumn{3}{|l|}{ Idade } \\
\hline 10 a 20 anos & 1 & $5 \%$ \\
\hline 41 a 50 anos & 2 & $10 \%$ \\
\hline 51 a 80 anos & 4 & $20 \%$ \\
\hline 61 a 70 anos & 12 & $60 \%$ \\
\hline 71 a 80 anos & 1 & $5 \%$ \\
\hline \multicolumn{3}{|l|}{ Sexo } \\
\hline Feminino & 20 & $100 \%$ \\
\hline \multicolumn{3}{|l|}{ Escolaridade } \\
\hline Ensino fundamental incompleto & 5 & $25 \%$ \\
\hline Ensino fundamental completo & 2 & $10 \%$ \\
\hline Ensino médio incompleto & 3 & $15 \%$ \\
\hline Ensino médio completo & 5 & $25 \%$ \\
\hline Ensino superior incompleto & 1 & $5 \%$ \\
\hline Ensino superior completo & 4 & $20 \%$ \\
\hline \multicolumn{3}{|l|}{ Renda } \\
\hline >1 a 2 salários mínimos & 8 & $40 \%$ \\
\hline >2 a 3 salários mínimos & 6 & $30 \%$ \\
\hline >3 a 4 salários mínimos & 2 & $10 \%$ \\
\hline >4 a 5 salários mínimos & 3 & $15 \%$ \\
\hline Não informou & 1 & $5 \%$ \\
\hline \multicolumn{3}{|l|}{ Estado civil } \\
\hline Casado & 11 & $55 \%$ \\
\hline Solteiro & 6 & $30 \%$ \\
\hline Viúvo & 2 & $10 \%$ \\
\hline Outros & 1 & $5 \%$ \\
\hline \multicolumn{3}{|l|}{ Grau de parentesco } \\
\hline Esposa & 5 & $25 \%$ \\
\hline Filha & 11 & $55 \%$ \\
\hline Neta & 1 & $5 \%$ \\
\hline Nora & 2 & $10 \%$ \\
\hline Outros & 1 & $5 \%$ \\
\hline
\end{tabular}

Fonte: elaborado pelos autores 
Observa-se que $60 \%$ dos respondentes possuem entre 61 e 70 anos de idade e $15 \%$ tem idade inferior a 50 anos. Todos os cuidadores são do sexo feminino, sendo que 35\% possuem o Ensino Fundamental (completo ou incompleto), 35\% o Ensino Médio (completo ou incompleto) e 25\%, o Ensino Superior (completo ou incompleto). Entre as participantes da pesquisa $55 \%$ declarara-se casados, $30 \%$ solteiros e $10 \%$ viúvos. Em relação ao grau de parentesco, um quarto dos cuidadores declararam-se casados com os idosos sob cuidados, $55 \%$ filhos destes idosos e $25 \%$ netos, noras ou possuem outro tipo de vínculo.

A Figura 1 apresenta a distribuição do escore do EZBI, instrumento utilizado para avaliar a percepção de sobrecarga de trabalho dos cuidadores. Verifica-se que a concentração dos escores está entre 20 e 40 pontos, sendo que metade dos respondentes obteve pontuação nesta faixa. Foi observado que o índice varia entre 23 e 72 pontos, com média e mediana de 44,7 e 40 pontos, respectivamente. Em relação à dispersão, obteve-se um desvio padrão de 16,18 pontos, com respectivo coeficiente de variação de $36,19 \%$, indicando uma dispersão razoável dos dados em torno da média.

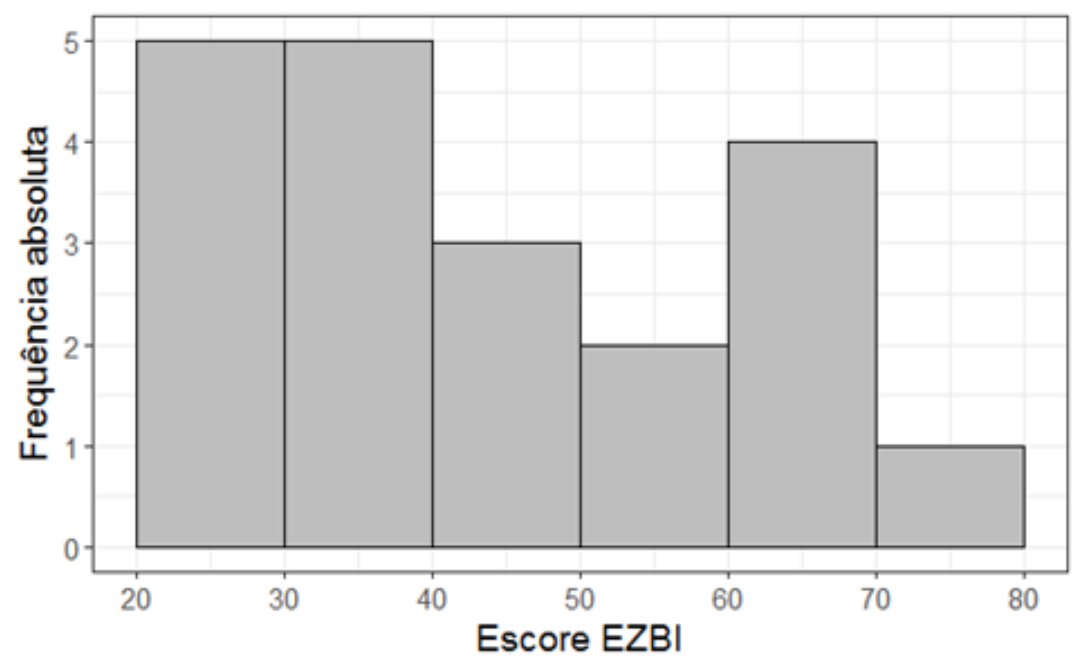

Figura 1 - Histograma do escore do EZBI obtido pelos cuidadores participantes da pesquisa. Fonte: Elaborado pelos autores

De acordo com o score estabelecido por Zarit e Zarit (1987), os resultados encontrados indicam que a sobrecarga dos cuidadores de idosos é moderada, visto que $50 \%$ obtiveram o escore de 21 a 40 pontos. $25 \%$ deles obtiveram escores compatíveis com sobrecarga moderada e severa, e $25 \%$ sobrecarga severa (Tabela 2). 
Tabela 2 - Escore de sobrecarga do cuidador de idoso

\begin{tabular}{llcc}
\hline Sobrecarga & Escore & Frequência & $\%$ \\
\hline Pequena & 0 a 20 pontos & 0 & $0 \%$ \\
Moderada & 21 a 40 pontos & 10 & $50 \%$ \\
Moderada a severa & 41 a 60 pontos & 5 & $25 \%$ \\
Severa & 61 a 88 pontos & 5 & $25 \%$ \\
\hline
\end{tabular}

Fonte: elaborado pelos autores

A Figura 2 apresenta as respostas para as questões do EZBI. Nessa apresentação, "S" refere-se ao idoso que é cuidado pelo entrevistado. Observase quando questionado se se sente envergonhado (a) com o comportamento do S (questão 4), sente que será incapaz de cuidar de S por muito mais tempo (questão 16) ou gostaria de simplesmente deixar que outra pessoa cuidasse de S (questão 18), a resposta predominante foi nunca, apontada por $60 \%, 45 \%$ e $60 \%$ dos respondentes, respectivamente. Nenhum dos cuidadores respondeu sempre para essas questões.

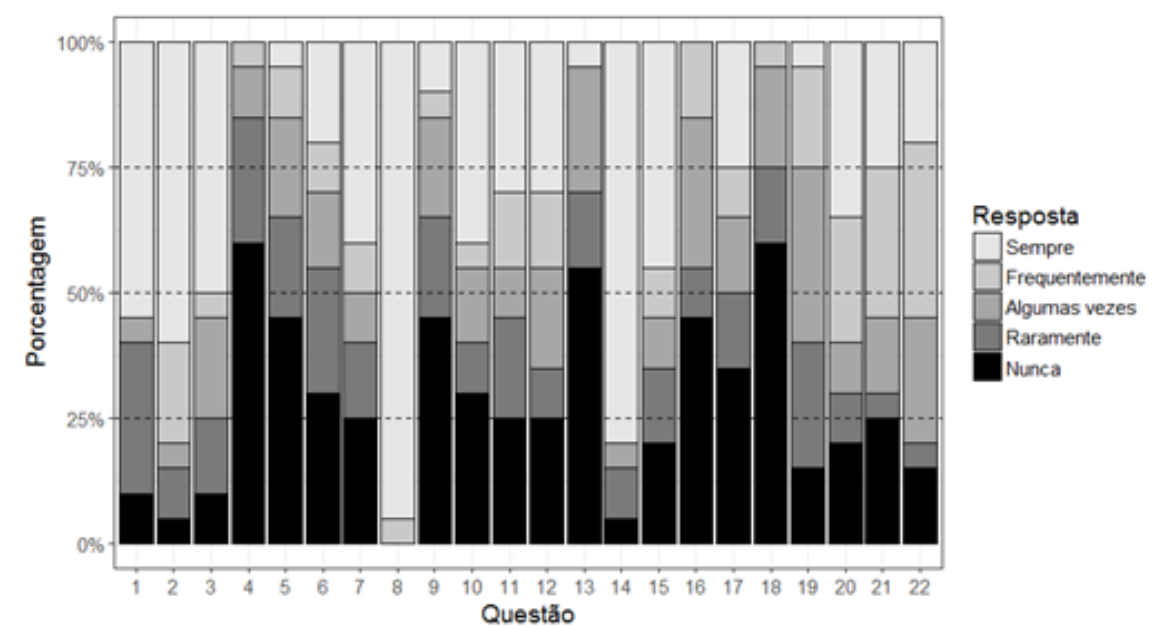

Figura 2 - Gráficos de barras empilhadas das respostas às 22 questões do EZBI, referente a sobrecarga relatadas pelos cuidadores participantes da pesquisa.

Fonte: elaborado pelos autores

${ }^{*} \mathrm{Na}$ questão 22, as respostas são nem um pouco, um pouco, moderadamente, muito extremamente, correspondendo respectivamente a nunca, raramente, algumas vezes, frequentemente e sempre.

Nunca foi a resposta mais frequente quando questionados se não se sente à vontade de ter visitas em casa, por causa de S (questão 13), se sente irritado (a) quando S está por perto (questão 5), se sente tenso (a) quando S está por perto (questão 9), sente que perdeu o controle da sua vida desde a doença de S (questão 17) ou sente que o S afeta negativamente seus relacionamentos com outros membros da família ou amigos (questão 6), uma vez que as mesmas foram relatadas por 55\%, 45\%, 45\%, 35\% e 30\% dos respondentes, para cada questão, respectivamente.

Por outro lado, 95\% dos participantes respondeu que sempre sente que S depende do Sr./Sr. a (questão 8). Destaca-se também que $80 \%$ dos cuidadores sentem que S espera que o Sr./Sra. cuide dele/dela, como se o Sr./Sr. fosse a única pessoa de quem ele/ela pode depender (questão 14), $60 \%$ sempre sentem 
que por causa do tempo que o Sr./Sr. ${ }^{\text {a }}$ gasta com o S, o Sr./Sr. ${ }^{a}$ não tem tempo suficiente para si mesma (questão 2), $55 \%$ sempre sentem que $\mathrm{S}$ pede mais ajuda do que ele (a) necessita (questão 1) e 50\% sempre se sentem estressadas entre cuidar do $\mathrm{S}$ e suas outras responsabilidades com a família e trabalho.

Verifica-se também que sempre é a resposta mais frequente às questões: $45 \%$ sempre sentem que não têm dinheiro suficiente para cuidar de $\mathrm{S}$, somando-se as suas outras despesas (questão 15), $40 \%$ sempre sentem receio pelo futuro de S (questão 7), 40\% sempre sentem que a sua saúde foi afetada por causa do seu envolvimento com S (questão 10), 35\% sempre sentem que deveriam estar fazendo mais por S (questão 20), 30\% sempre sentem que o Sr./Sr. ${ }^{a}$ não têm tanta privacidade como gostariam, por causa de S (questão 11) e 30\% sempre sentem que a sua vida social tem sido prejudicada porque o Sr./Sra ${ }^{\underline{a}}$ está cuidando de S (questão 12).

Quando questionados se sentem em dúvida sobre o que fazer por S (questão 19), $35 \%$ dos cuidadores responderam algumas vezes, enquanto que $30 \%$ frequentemente sentem que poderiam cuidar melhor de $S$ (questão 21). Por fim, de uma maneira geral, $35 \%$ dos cuidadores apontam que se sentem muito sobrecarregados (as) por cuidar de S (questão 22), enquanto $20 \%$ sentem-se nem um pouco ou um pouco sobrecarregados.

Observa-se na Figura 3, que apesar da grande dispersão do escore EZBI, a pontuação parece aumentar de acordo com a faixa de idade dos respondentes, apresentando um coeficiente de correlação de Spearman de 0,26. Quanto à escolaridade, nota-se uma pontuação alta no EZBI para os cuidadores com ensino fundamental incompleto, pontuações baixas foram observadas para aqueles com ensino fundamental completo e ensino médio incompleto. Visualmente, não há uma relação clara de aumento ou diminuição do EZBI de acordo com a escolaridade, sendo que o coeficiente de correlação de Spearman calculado entre as duas variáveis foi de $-0,18$. Por outro lado, observa-se uma tendência de diminuição do escore EZBI com o aumento da faixa de renda dos cuidadores, sendo que o coeficiente de correlação de Spearman entre tais variáveis foi de $-0,22$.

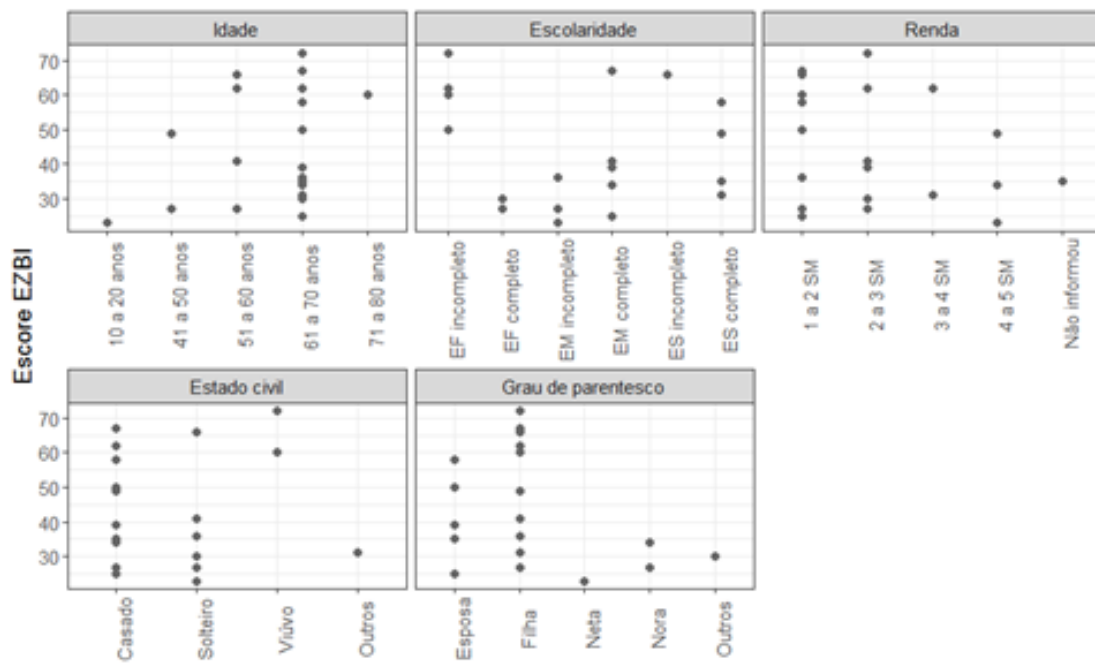

Figura 3 - Diagrama de dispersão entre o escore do EZBI e as variáveis sociodemográficas dos cuidadores participantes da pesquisa.

Fonte: elaborado pelos autores 
Comparando os escores do EZBI dos cuidadores cujo estado civil corresponde a casado ou solteiro, obteve-se um coeficiente $D$ de Somer de -0,32, indicando que a pontuação é menor entre os solteiros em relação aos casados, corroborando com o observado na Figura 3. Ainda, nota-se que a pontuação dos cuidadores cujo grau de parentesco com o idoso é filha, aparenta ser um pouco maior em relação às esposas, apresentando um coeficiente $D$ de Somer de 0,09.

\section{DISCUSSÃO}

Os principais resultados deste estudo, baseado em uma amostra de cuidadores informais de idosos atendidos pelo programa ESF, são: a maioria dos cuidadores demonstraram ter uma percepção de sobrecarga de trabalho moderada; esta percepção tem relação com condições socioeconômicas e demográficas, sendo que aqueles cuidadores com idade mais avançada, menor grau de escolaridade, menor renda familiar e casados apresentam maior sobrecarga de trabalho; as dificuldades no cotidiano de desempenho do encargo que transparecem na observação das falas são amenizadas pelos laços afetivos estabelecidos no ambiente de trabalho.

As características dos cuidadores observadas no presente estudo se assemelham às descritas em outros estudos, tanto nacionais quanto internacionais, em que a maioria dos cuidadores informais de idosos é do sexo feminino, com idade superior a 50 anos e sem ensino superior (SILVA LEITE et al., 2017; DOS SANTOS-ORLANDI et al., 2017; GUTIERREZ; FERNANDES; MASCARENHAS, 2017; EBY et al., 2017) sugerindo que mesmo se tratando de uma amostra pequena e regionalizada, as características sociodemográficas pouco se alteram nesta população. Ademais, utilizando a escala de sobrecarga por meio do EZBI, notamos que a maioria dos cuidadores apresentaram escores compatíveis com carga de trabalho moderada, o que também foi observado em outros estudos, realizados tanto no exterior quanto no Brasil, utilizando esta mesma escala (RINGER et al., 2016; VAINGANKAR et al., 2016; ALSHAMMARI et al., 2017; AJAY, et al., 2017.; MELLO et al., 2017), fortalecendo a hipótese os resultados da presente pesquisa pode ser representativa da população de cuidadores de idosos, pelo menos no que se restringe a análise da percepção de sobrecarga de trabalho.

A faixa etária dos cuidadores é uma variante de relevância, visto que, muitas vezes o cuidador pertence a mesma geração de indivíduos cuidados, neste caso, idosos independentes que cuidam de idosos dependentes. A correlação positiva entre idade e percepção de sobrecarga de trabalho demonstrada no presente estudo, indica que quanto mais avançada a idade, maior a sobrecarga. Esta constatação pode ser atribuída tanto a limitações físicas inerentes ao envelhecimento (DOS SANTOS-ORLANDI et al., 2017; AJAY, et al., 2017. MELLO et al., 2017), quanto com aspectos psíquicos em detrimento do convívio mais intenso com o idoso, visto que cuidadores mais jovens possuem uma vida social mais ativa (CHEN et al., 2017). Outra condição relativa a sobrecarga dos cuidadores está associada às dificuldades financeiras e baixo grau de instrução, uma vez que se observou que cuidadores com menor renda e menor escolaridade apresentaram maiores escores na escala EZBI, assinalando que os desdobramentos negativos da vulnerabilidade social dos cuidadores amplificam sua percepção de sobrecarga de trabalho. Associação positiva entre ser casado e sobrecarga de trabalho foi também observada em nosso estudo, o 
que pode ser explicado pelo fato de que os cônjuges frequentemente manifestam maior angústia e sentimentos de sobrecarga quando eles sozinhos assumem o cuidado de um idoso em casa (VAINGANKAR et al., 2016; DE VUGT et al., 2006).

A se indagar sobre possibilidade de a própria saúde ser afetada devido ao envolvimento com o idoso, observa-se respostas que envolvem aspectos subjetivos conforme demonstrado em fala: "Meu emocional fica muito sobrecarregado, às vezes a gente chora calado para não extravasar" (LMM). Muitos cuidadores identificaram que sua saúde foi afetada por causa do envolvimento com o idoso, tanto em aspectos físicos como psicossociais, o que demostra uma percepção consciente das consequências subjetivas da sobrecarga de trabalho. De fato, há registros de que tal sobrecarga repercute drasticamente na saúde do cuidador e o condiciona a vulnerabilidade (SILVA LEITE et al., 2017; DE VALLE-ALLONSO et al., 2015). Além disso, a fragilidade física do idoso tem sido associada com aumento da percepção de sobrecarga de trabalho do cuidador (RINGER et al., 2016), uma vez que muitos cuidadores deixam de realizar o cuidado com a própria saúde e passam a priorizar o cuidado do idoso. Este fato foi também revelado nas análises de conteúdo das falas, conforme relato exemplo de DMZ: "Agora a gente vai mais para o hospital para levar ela, deixa de cuidar da gente. Eu até falei para a minha vizinha que já faz dois anos que não faço uma mamografia" (MLS).

O fato de a maioria dos cuidadores responderem nunca quando questionados se gostariam de simplesmente deixar que outra pessoa cuidasse do idoso sob sua responsabilidade, sugere que mesmo diante de uma sobrecarga de trabalho o vínculo emocional entre cuidador e o cuidado parece amenizar as dificuldades do ato de cuidar. Notamos nas falas um predomínio de aspectos relacionados à gratidão, responsabilidade e o amor pelo idoso, conforme narrado: "Pai e Mãe é precioso, eu sou mãe e não fazemos mais que o dever de cuidar bem dos nossos pais e avós. Meu pai já se foi e agora eu só tenho ela" (JRS). Interessantemente, em estudo realizado por Rodrigues et al. (2014), os cuidadores de idosos identificaram sua saúde como regular e descreveram piora quando comparada ao período em que não exerciam tal função, fortalecendo a hipótese de que os laços emocionais interferem na análise da sobrecarga de trabalho.

O elevado grau de dependência e a consciência dos cuidadores de que são as únicas pessoas com as quais contam o idoso pode também ser identificada na análise qualitativa das entrevistas dos cuidadores. Conforme narrado "Quando eu saio de casa para fazer alguma coisa, ela já fica perguntando por mim, eu acho que é porque ela se sente mais segura quando eu estou por perto, não confia nos outros para cuidar dela" (ARC). No mesmo sentido, quando questionados a respeito da existência de outras pessoas que ajudam a cuidar do idoso para que os cuidadores possam realizar suas atividades pessoais, a maioria deles respondeu que entes do próprio núcleo familiar ou até mesmo vizinhos acabam ficando com o idoso por curto período de tempo, em situações de extrema necessidade. Contudo, na grande parte dos dias e na maioria das vezes, a responsabilidade do cuidado recai somente sobre o cuidador, segundo relatado "Eu sou a única pessoa pra cuidar dele, por isso peço para Deus dar paciência e força pra eu cuidar dele até o fim" (TMO).

Durante as entrevistas destes cuidadores verificou-se que a maioria dos cuidadores apontou que sente falta de realizar atividades de lazer, como descrito: "Eu tenho vontade de ir para igreja, fazer caminhadas, meus cursos, colocar a minha cabeça para funcionar. Mas não tem como" (LMM). Foram também relatadas vontade de viajar com a família, ir ao cinema, fazer exercício, 
trabalhar para ter a própria renda, cuidar de si, visitar amigos e familiares, atividades que foram comprometidas devido a responsabilidade com os idosos. A escala ZBI revela-se interessante para a aplicação a cuidadores de idosos a fim de subsidiar associações relevantes entre altos graus de sobrecarga, principalmente quando se planeja ações de enfrentamento das dificuldades laborais deste cenário peculiar de vulnerabilidade (FREDERICK, 2018; BIANCHI et al, 2016)

\section{CONSIDERAÇÕES FINAIS}

Os resultados da presente pesquisa apontam a relevância dos determinantes sociais de saúde dos cuidadores para a percepção da sobrecarga de trabalho. Da mesma forma, as relações fraternas desenvolvidas ao longo do processo do cuidar também interferem na percepção dessa sobrecarga. Assim, infere-se que são cada vez mais necessárias discussões acerca dos determinantes sociais de saúde dos cuidadores informais e suas implicações para o delineamento de estratégias promotoras de saúde específicas para essa população.

De forma geral, nosso estudo contribui para melhorar o conhecimento acerca das situações que envolvem a atuação do cuidador informal de idosos, focando nos fatores que estão associados a percepção de sobrecarga de trabalho por estes cuidadores. A análise dos resultados encontrados de escore moderado de sobrecarga permite pressupor que as dificuldades no cotidiano de desempenho do encargo que transparecem na observação das falas são amenizadas pelos laços afetivos estabelecidos entre os cuidadores e os idosos.

\section{REFERÊNCIAS}

ABAJOBIR, A. A. et al. Global, regional, and national disability-adjusted lifeyears (DALYs) for 333 diseases and injuries and healthy life expectancy (HALE) for 195 countries and territories, 1990-2016: a systematic analysis for the Global Burden of Disease Study 2016. Lancet, v.390, p.1260-344, 2017. Disponível em < https://www.thelancet.com/journals/lancet/article/PIIS01406736(17)31833-0/abstract\#\%20> Acesso em: 19 st. 2018.

AJAY, Shweta eta al., Association of impairments of older persons with caregiver burden among family caregivers: Findings from rural South India. Archives of Gerontology and Geriatrics, v.68, p.143-148, 2017. Disponível em: < https://www.sciencedirect.com/science/article/pii/S0167494316301832> Acesso em 19 set. 2018.

ALSAEED, D. et al. Challenges to optimal medicines use in people living with dementia and their caregivers: A literature review. International Journal of Pharmaceutics, v.512, n.2, p.396-404, 2016. Disponíve em:< https://www.sciencedirect.com/science/article/pii/S0378517315304488?via\%3D ihub> Acesso em 19 set. 2018.

ALSHAMMARI, Sulaiman A. et al. The burden perceived by informal caregivers of the elderly in Saudi Arabia. Journal of Family Community Medicine, v.24, n.3, p.145-150, 2017. Disponível em <https://www.ncbi.nlm.nih.gov/pmc/articles/PMC5596626/> Acesso em: 18 set. 2018. 
BATISTA, Marina Picazzio Perez; ALMEIDA, Maria Helena Morgani de; LANCMAN, Selma. Cuidadores formais de idosos: contextualização histórica no cenário brasileiro. Revista Brasileira de Geriatria e Gerontologia. Rio de Janeiro, v. 17, n. 4, p. 879-885. Disponível em: http://www.scielo.br/scielo.php?script=sci_arttext\&pid=S180998232014000400879\&Ing=en\&nrm=iso> Acesso em 19 set. 2018.

BIANCHI, Mariana et. al. Zarit Burden Interview Psychometric Indicators Applied in Older People Caregivers of Other Elderly. Revista Latino-Americana de Enfermagem, v.24, 2016. Disponível em: <

http://www.scielo.br/scielo.php?script=sci_arttext\&pid=S0104$11692016000100433>$ Acesso em 19 set. 2018.

BORN, Tomiko. Cuidar Melhor e Evitar a Violência. Manual do Cuidador da Pessoa Idosa. Brasília: Secretaria Especial dos Direitos Humanos, 2008.

BRASIL. Ministério da Previdência e Assistência Social. Portaria Interministerial MS/MPAS № 5.153, de 7 de abril de 1999. Programa Nacional de Cuidadores de Idosos. 1999.

. Ministério da Saúde. Portaria № 2.528 De 19 De Outubro De 2006 Aprova a Política Nacional de Saúde da Pessoa Idosa. 2006.

CHEN, Ming Chun et al. Effects of home-based long-term care services on caregiver health according to age. Health Qual Life Outcomes, v.15, n.1, p:110, 2017. Disponível em < https://www.ncbi.nlm.nih.gov/pmc/articles/PMC5651602/> Acesso em 19 set. 2018.

COMMISSO, Elana et al. Identifying and understanding the health and social care needs of older adults with multiple chronic conditions and their caregivers: a protocol for a scoping review, BMJ Open v.7, n.12, 2017. Disponível em < https://bmjopen.bmj.com/content/7/12/e018247> Acesso em 19 set. 2018.

DE VALLE-ALONSO M. J. et al. Sobrecarga y Burnout en cuidadores informales del adulto mayor. Enfermería Univiversitaria, v.12, n.1, 2015, p.19-27. Disponível em <http://www.scielo.org.mx/pdf/eu/v12n1/v12n1a4.pdf > Acesso em 19 set. 2018

DE VUGT, Marjolein E. et al. Impact of behavioural problems on spousal caregivers: a comparison between Alzheimer's disease and frontotemporal dementia. Dementia and Geriatric Cognitive Disorders, v.22, n.1, p.35-41, 2006. Disponível em: <<https://www.ncbi.nlm.nih.gov/pubmed/16679763> Acesso em: 18 set. 2018.

DEBERT, Guita Grin; OLIVEIRA, Amanda Marques de. A profissionalização da atividade de cuidar de idosos no Brasil. Revista Brasileira de Ciência Política, Brasília, n. 18, p. 7-41, 2015. Disponível em: http://www.scielo.br/scielo.php?script=sci_arttext\&pid=S0103$33522015000400007 \&$ Ing=en\&nrm=iso> Acesso em 19 set. 2018.

DEL-PINO-CASADO, Rafael et al. Social support and subjective burden in caregivers of adults and older adults: A meta-analysis. PLoS One, v.13, n1, p.1-18, 2018. Disponível em: < https://journals.plos.org/plosone/article?id=10.1371/journal.pone.0189874> Acesso em 19 set. 2018. 
DOS SANTOS-ORLANDI Ariene Angelini et alldosos que cuidam de idosos: um estudo sobre a Síndrome da Fragilidade. Revista Brasileira de Enfermagem, v.70, n.4, p.856-864, 2017. Disponível em: <http://www.redalyc.org/html/2670/267052023022/> Acesso em 19 set. 2018.

EBY, David W. et al. Characteristics of informal caregivers who provide transportation assistance to older adults. PLoS One, v. 12, n.9, p.1-14, 2017. Disponível em < https://www.ncbi.nlm.nih.gov/pmc/articles/PMC5607132/> Acesso em 18 set. 2018.

FEKETE, Christiane et al. Health impact of objective burden, subjective burden and positive aspects of caregiving: an observational study among caregivers in Switzerland. BMJ Open, v.7, n.12, 2017. Disponível em <https://bmjopen.bmj.com/content/7/12/e017369> Acesso em 19 de set. 2018.

FREDERICK, D. Mitigating Burden Associated with Informal Caregiving. Journal of Patient Experience. V.5, n.1, p.50-5, 2018. Disponível em < https://www.ncbi.nlm.nih.gov/pmc/articles/PMC5862383/> Acesso em 19 de set. 2018.

GUTIERREZ, Lucila Ludmila Paula; FERNANDES, Neisa Rejane Machado; MASCARENHAS, Marcello. Caracterização de cuidadores de idosos da região metropolitana de Porto Alegre (RS): perfil do cuidado. Saúde em Debate, v. 41, n. 114, p. 885-898, 2017. Disponível em < http://www.scielo.br/scielo.php?script=sci_arttext\&pid=S0103$11042017000300885 \&$ Ing=en\&nrm=iso, Acesso em 19 set. 2018.

KOYANAGI, A. et al. Depression, sleep problems, and perceived stress among informal caregivers in 58 low-, middle-, and high-income countries: A crosssectional analysis of community-based surveys. Journal of Psychiatric Research, v.96, p. 115-23, 2018. Disponível em <https://www.ncbi.nlm.nih.gov/pubmed/29031131> Acesso em: 19 set. 2018.

LAMBERT, Sylvie D. et al. Impact of informal caregiving on older adults' physical and mental health in low-income and middle-income countries: a cross-sectional, secondary analysis based on the WHO's Study on global AGEing and adult health (SAGE). BMJ Open, v.7, n.11, 2017. Disponível em $<$ https://bmjopen.bmj.com/content/7/11/e017236 > Acesso em 19 set. 2018.

LIMA-COSTA, Maria Fernanda et al. Cuidado informal e remunerado aos idosos no Brasil (Pesquisa Nacional de Saúde, 2013). Revista de Saúde Pública, São Paulo, v. 51, supl. 1, 6s, 2017. Disponível em: http://www.scielo.br/scielo.php?pid=S003489102017000200311\&script=sci_artt ext\&tlng=pt>. Accesso em 19 set 2018.

LIMA-COSTA, Maria Fernanda et al. Socioeconomic inequalities in activities of daily living limitations and in the provision of informal and formal care for noninstitutionalized older Brazilians: National Health Survey, 2013. International Journal of Equity in Health. v.15, n.1, p.1-8, 2016. Disponível em: < https://www.ncbi.nlm.nih.gov/pmc/articles/PMC5112736/> Acesso em 19 set. 2018.

LÓPEZ-MARTÍNEZ, Catalina; Frías-Osuna Antonio; Del-Pino-Casado Rafael. Sentido de coherencia y sobrecarga subjetiva, ansiedad y depresión en personas cuidadoras de familiares mayores. Gaceta Sanitaria, 2017. Disponível em: < 
https://www.sciencedirect.com/science/article/pii/S0213911117302601> Acesso em 19 de set. 2018.

MADARA, Marasinghe K. Assistive technologies in reducing caregiver burden among informal caregivers of older adults: a systematic review. Disability and Rehabilitation: Assistive Technology, v.11, n.5, p.353-360, 2016. Disponível em < https://www.ncbi.nlm.nih.gov/pubmed/26371519> Acesso em 19 set. 2018.

MELLO, Johanna A. et al. The determinants of informal caregivers' burden in the care of frail older persons: a dynamic and role-related perspective. Aging Mental Health, v. 21, n.8, p.838-843, 2017. Disponível em

<https://www.ncbi.nlm.nih.gov/pubmed/27054404> Acesso em: 18 set. 2018.

MONTEIRO, Edilene Araújo; MAZIN, Suleimy Cristina; DANTAS, Rosana Aparecida Spadoti. Questionário de Avaliação da Sobrecarga do Cuidador Informal: validação para o Brasil. Revista Brasileira de Enfermagem, v. 68, n 3, p. 421-428, 2015. Disponível em

http://www.scielo.br/scielo.php?script=sci_arttext\&pid=S0034-

$71672015000300421 \&$ Ing=en\&nrm=iso > Acesso em 19 set. 2018.

MOTA, Fernanda Rochelly do Nascimento et al. Cross-cultural adaptation of the Caregiver Reaction Assessment for use in Brazil with informal caregivers of the elderly. Revista da Escola de Enfermagem da USP, v. 49, n. 3, p. 424431, 2015 Disponível em

http://www.scielo.br/scielo.php?script=sci_arttext\&pid=S0080-

$62342015000300424 \&$ Ing=en\&nrm=iso> Acesso em 19 set. 2018.

MUNIZ, Emanoel Avelar et al. Grau de sobrecarga dos cuidadores de idosos atendidos em domicílio pela Estratégia Saúde da Família. Saúde em Debate. V.40, n.110, p.172-182. 2016. Disponível em: http://www.scielo.br/pdf/sdeb/v40n110/0103-1104-sdeb-40-110-0172.pdf> Acesso em: 19 set. 2018.

OLDENKAMP, Marloes et al. The impact of older person's frailty on the carerelated quality of life of their informal caregiver over time: results from the TOPICS-MDS project. Quality Life of Research, v.26, n.10, p.2705-2716, 2017. Disponível em < https://www.ncbi.nlm.nih.gov/pubmed/28567602> Acesso em 19 set. 2018.

OLDENKAMP, Marloes et al. Subjective burden among spousal and adult-child informal caregivers of older adults: results from a longitudinal cohort study. BMC Geriatrics. V.16, n.1, p.1-11, 2016. Disponível em < https://www.ncbi.nlm.nih.gov/pmc/articles/PMC5142272/> > Acesso em 19 set. 2018.

PENDERGRASS, A. et al. Screening for caregivers at risk: Extended validation of the short version of the Burden Scale for Family Caregivers (BSFC-s) with a valid classification system for caregivers caring for an older person at home. BMC Health Services Research, v.18, n.1, p.229, 2018. Disponível em <https://www.ncbi.nlm.nih.gov/pubmed/29609600> Acesso em 19 set. 2018.

RINGER, Thom J. et al. Care recipients' physical frailty is independently associated with subjective burden in informal caregivers in the community setting: a cross-sectional study. BMC Geriatrics, v.16, n.1, p.1-5, 2016. 
Disponível em < https://www.ncbi.nlm.nih.gov/pmc/articles/PMC5114771/ > Acesso em 19 set. 2018.

RODRIGUES, J. E. G. et al. Qualidade de vida e sobrecarga de cuidadores familiares de idosos dependentes. Ciência y Enfermeria, v. 20, n. 3, p.119129, 2014. Disponível em:< https://scielo.conicyt.cl/pdf/cienf/v20n3/art_11.pdf> Acesso em 19 set. 2018.

SANDERSON, W. C.; SCHERBOV, Sergei.; GERLAND, Patrick. Probabilistic population aging. PLoS One. 2017; 12(6):0179171.

SCAZUFCA, Marcia. Brazilian version of the Burden Interview scale for the assessment of burden of care in carers of people with mental illnesses. Revista Brasileira de Psiquiatria, v. 24, n. 1, p. 12-17, 2002. Disponível em< $<$ http://www.scielo.br/scielo.php?script=sci_arttext\&pid=S1516$44462002000100006 \&$ Ing=en\&nrm=iso > Acesso em 19 set. 2018.

SILVA LEITE Bruna et. al. A vulnerabilidade dos cuidadores de idosos com demência: estudo descritivo transversal. Revista Brasileira de Enfermagem, v.70, n.4, p.714-720, 2017. Disponível em: <

http://www.scielo.br/pdf/reben/v70n4/pt_0034-7167-reben-70-04-0682.pdf> Acesso em 19 set. 2018.

SINGER, M. A. The Origins of Aging: Evidence that Aging is an Adaptive Phenotype. Current Aging Science, v.9, n.2, p.95-115, 2016.

TKATCH, Rifky et al. A Pilot Online Mindfulness Intervention to Decrease Caregiver Burden and Improve Psychological Well-Being. Journal of Evidence-Based Complementary and Alternative Medicine, v.22, n.4, p.736-43, 2017.Disponível em < https://www.ncbi.nlm.nih.gov/pubmed/29228806> Acesso em 19 set. 2018.

TKATCH Rifky et al. Population Health Management for Older Adults. Gerontology and Geriatric Medicine, v.2, 2016. Disponível em: < https://www.ncbi.nlm.nih.gov/pmc/articles/PMC5486489/> Acesso em: 19 set 2018.

VAINGANKAR, Janhavi Ajit et al. Care participation and burden among informal caregivers of older adults with care needs and associations with dementia. International Psychogeriatrics, v.28, n.2, p.221-231. Disponível em < https://www.ncbi.nlm.nih.gov/pubmed/26478530> Acesso em 19 set. 2018.

WANG, Haidong et al. Global, regional, and national under-5 mortality, adult mortality, age-specific mortality, and life expectancy, 1970-2016: a systematic analysis for the Global Burden of Disease Study 2016. Lancet, v.390, p.1084150, 2017.

WHO. World Health Organization. World Population Ageing. Highlights Global strategy and action plan on ageing and health. New York: United Nations, Department of Economic and Social Affairs Population Division. New York, 2017. Disponível em:

<http://www.un.org/en/development/desa/population/publications/pdf/ageing/W PA2017_Highlights.pdf> Acesso em: 18 set. 2018.

World Health Organization. World Report on Ageing and Health.

Genebra: World Health Organization, 2015. Disponível em:< 
http://www.who.int/ageing/events/world-report-2015-launch/en/> Acesso em 18 set. 2018 .

ZARIT S.; ZARIT J. Instructions for the Burden Interview. University Park: Pennsylvania State University; 1987. Disponível em <

http://dementiapathways.ie/_filecache/edd/c3c/89-zarit_burden_interview.pdf> Acesso em 19 set. 2018

Recebido em: 24-09-2018

Aceito em: 25-05-2020 